Cahiers $d u$ MONDE RUSSE

\section{Cahiers du monde russe}

Russie - Empire russe - Union soviétique et États indépendants

50/2-3 | 2009

L'Europe orientale, 1650-1730. Crises, conflits et renouveau

\title{
Le Droit de propriété en mutationAurore
} CHAIGNEAU

\author{
, Paris : Dalloz, 2008, 683 p.
}

Jessica Allina-Pisano

\section{OpenEdition}

\section{Journals}

\section{Édition électronique}

URL : https://journals.openedition.org/monderusse/9789

DOI : 10.4000/monderusse. 9789

ISSN : $1777-5388$

\section{Éditeur}

Éditions de l'EHESS

\section{Édition imprimée}

Date de publication : 15 septembre 2009

ISBN : 978-2-7132-2260-3

ISSN : $1252-6576$

\section{Référence électronique}

Jessica Allina-Pisano, « Le Droit de propriété en mutationAurore CHAIGNEAU », Cahiers du monde russe [En ligne], 50/2-3 | 2009, mis en ligne le 14 janvier 2013, consulté le 03 septembre 2022. URL http://journals.openedition.org/monderusse/9789; DOI : https://doi.org/10.4000/monderusse.9789

Ce document a été généré automatiquement le 3 septembre 2022

Tous droits réservés 


\section{Le Droit de propriété en mutationAurore CHAIGNEAU}

, Paris : Dalloz, 2008, 683 p.

Jessica Allina-Pisano

\section{Aurore CHAIGNEAU, Le Droit de propriété en mutation, Essai à la lumière du droit russe, Paris : Dalloz, 2008, 683 p.}

Dans cette analyse minutieuse de la théorie du droit en Russie, Aurore Chaigneau nous expose le cheminement de l'idée et de la pratique de la propriété en Russie pendant plus de deux siècles. L'ouvrage présente un récit historique culminant dans une discussion des solutions possibles en matière de droit à la gestion, la maîtrise et l'appropriation des biens complexes, particulièrement les ressources naturelles.

2 Cet approfondissement est particulièrement bienvenu dans un contexte global de redéfinition, fragmentation et marchandisation des biens publics. De plus, dans le contexte russe d'aujourd'hui, après le pillage et l'abandon de ces biens pendant la période de privatisation et les efforts récents du gouvernement pour se les réapproprier, cette réflexion juridique tombe fort à propos. La couverture très exhaustive du développement de la philosophie du droit dans ce pays, les débats entre penseurs russes, l'attention portée à la pratique de la jurisprudence dans un contexte de profondes transformations sociales, économiques et politiques, nous valent une contribution significative aux études du droit et de l'histoire russe en Occident.

Ce livre est exceptionnel par la qualité et la quantité de preuves fournies par l'auteur. Son argumentation repose sur une grande variété de sources, traitées de façon méticuleuse et cumulative. En particulier, l'utilisation extensive d'exemples tirés de la jurisprudence et une approche partagée avec d'autres spécialistes du même champ produisent un livre fascinant tant pour ceux qui s'intéressent au droit ou à l'histoire russe que pour les politologues et les sociologues. Bien que l'ouvrage n'aborde pas tous les enjeux politiques ayant une incidence sur le développement du droit, la recension détaillée de documents de tribunaux ouvre au lecteur une fenêtre sur la réalité de la 
pratique du droit de la propriété en Russie et en Union soviétique. Cette approche présente cependant des limites, puisqu'elle n'observe la pratique du droit que dans les cas de contentieux, où les parties disposent de ressources sociales et matérielles suffisantes pour défendre leurs intérêts devant une cour. Toutefois, contrairement aux approches reposant uniquement sur la théorie du droit, Chaigneau plonge le lecteur dans la vie des gens, là où le droit prend son sens social.

4 L'auteur multiplie les contextes : Russie impériale, socialiste, postsocialiste, procédant de façon non chronologique. Elle examine successivement les droits soviétiques de la propriété, le droit libéral du XIX ${ }^{e}$ siècle et des dernières années du règne des Romanov, enfin la répartition de la propriété après la chute du communisme. Cette approche sert l'analyse des transformations postsoviétiques en soulignant l'influence de la pensée de l'époque impériale dans les débats sur la privatisation à la fin $\mathrm{du} \mathrm{xx}^{\mathrm{e}}$ siècle. On pourrait cependant estimer que l'organisation du livre, influençant le déroulement de l'analyse, dissimule autant qu'elle éclaire: l'exégèse du droit entre 1830 et 1917 inclut non seulement les débats pertinents sur les enjeux de la période postsoviétique, mais aussi bien d'autres réflexions qui ne furent pas impliquées dans les débats des années 1990, et qui sont intéressantes surtout pour les historiens de la Russie impériale.

5 La contribution majeure de ce livre réside dans son analyse approfondie du développement de la philosophie du droit en Russie. Une recension de quelques pages ne peut pas rendre justice à une analyse érudite, magistrale et détaillée de l'histoire des régimes de propriété, pas plus qu'elle ne peut élucider de manière adéquate les tensions complexes entre les concepts de biens collectifs et les propriétés collectives en pratique. Limitons-nous donc à deux questions d'intérêt particulier que soulève, explicitement ou implicitement, l'argumentation du livre : l'instrumentalisation du droit en Russie et ses implications pour la société; la question de la normativité dans l'analyse du développement institutionnel.

6 Aurore Chaigneau décèle les racines historiques de la dualité fondamentale de la société russe postsocialiste: existence d'un système de droit qui gère la relation entre l'État et ses sujets, pratiques officieuses qui arbitrent les relations entre individus au sein de l'État et dans la société. L'auteur identifie les origines de l'existence parallèle du droit officiel et du droit coutumier dans la période impériale et montre la continuation de cette dichotomie à travers la période socialiste.

7 Alors que certains auteurs interprètent cette divergence comme une preuve du caractère superflu ou inadéquat du droit en Russie, Chaigneau, a contrario, refuse d'écarter le droit et lui rend l'importance primordiale qu'elle lui accorde. C'est ce qui explique la contribution novatrice du livre pour la compréhension de la période soviétique. Celui-ci prend au sérieux l'importance de la pensée du droit soviétique en tant qu'idée fondatrice de la vision soviétique du monde et de l'économie soviétique. Contrairement aux analyses qui ne voient dans le droit soviétique qu'un «tigre de papier», cet ouvrage démontre la centralité du droit dans le système politique et économique soviétique. Le droit soviétique n'est pas une abstraction qui ne correspond pas à la réalité, mais un moteur incontournable du développement (p. 180-181).

8 La puissance de ce moteur provient de son instrumentalisation. Pour l'auteur, l'instrumentalisation du droit par l'État fonde la nature même de la propriété dans le contexte soviétique : le droit de la propriété, comme toute autre institution soviétique, n'existait qu'en tant qu'instrument pour créer des citoyens socialistes, refaire l'homme à l'image du travailleur et du consommateur socialiste et non pour encadrer la justice telle 
qu'elle est conceptualisée dans les sociétés libérales. En d'autres termes, le droit n'acquiert toute son importance qu'à travers son instrumentalisation par l'État. Si, dans les sociétés libérales occidentales, le droit est un instrument au service du bien-être des individus, dans le contexte russe, c'est un instrument de reproduction du pouvoir étatique.

Bien que l'auteur partage ici le point de vue d'autres spécialistes sur l'instrumentalisation du droit par le régime soviétique, son interprétation n'en est pas moins originale. Ainsi, Kathryn Hendley, auteur de plusieurs ouvrages sur le droit soviétique et russe, voit précisément dans l'instrumentalisation du droit par l'État la source de la non-pertinence du droit dans la société soviétique. Dans la logique du marxisme-léninisme, les masses inconscientes sont exclues $d u$ processus $d u$ développement du droit; elles sont destinées à obéir à la loi, non à la définir. Cette exclusion produit l'indifférence de la population vis-à-vis du droit. En revanche, pour Chaigneau, le pouvoir normatif de la conceptualisation du droit de propriété en tant qu'obligation - « devoir de », au lieu de « droit de » (p. 84) - contribue à l'importance du droit dans la société; le droit agit en tant qu'institution fondatrice de l'ordre soviétique, c'est le levier du développement et de la réforme sociale.

La description des mécanismes de l'instrumentalisation du droit par l'État soviétique nous vaut des illustrations colorées et saisissantes qui rendent le sujet vivant. Ainsi, les tribunaux soviétiques et postsoviétiques protégeaient surtout les règles administratives de la société et non les intérêts des citoyens en tant que tels. D'où l'exemple de l'histoire des portes métalliques installées à l'envers dans certains immeubles collectifs. Parfois, ces portes qui ouvraient vers l'extérieur empêchaient les voisins d'ouvrir les portes de leurs propres appartements. Les tribunaux statuent: l'installation de portes à l'envers constitue une infraction aux règlements de construction. Selon l'auteur, il ne s'agit nullement d'une contravention aux droits des individus, mais d'une défaillance administrative qu'il faut régler pour satisfaire les besoins de l'État.

L'instrumentalisation du droit au sein du système soviétique soulève selon nous une deuxième série d'interrogations liées à la normativité dans l'analyse comparative du droit. Le droit, comme tout ce qui touche aux questions politiques relatives aux périodes soviétique et postsoviétique, est parfois idéologisé, biaisé par les héritages discursifs de la guerre froide. Chaigneau aborde le sujet d'une façon relativement neutre. Ceci dit, malgré sa méthodologie stricte, l'analyse n'échappe pas à la normativité libérale. Le lecteur a parfois l'impression que certains systèmes de droit sont plus normatifs que d'autres. L'explication de la logique du rôle de la propriété dans la théorie du droit socialiste suggère une subjectivité particulière au sein du droit soviétique. La propriété dans le contexte de l'URSS existe pour satisfaire les besoins de l'homme socialiste, or ces besoins sont déterminés par les planificateurs de l'État soviétique.

Bien que l'auteur reconnaisse l'instrumentalisation du droit dans les régimes libéraux ( $\mathrm{p}$. 249), cet aspect reste relativement inexploré dans l'ouvrage. Pour certains auteurs, même si les besoins humains servis par le droit dans les sociétés libérales ne sont pas des créations directes des élites étatiques, une normativité implicite et une approche descendante de la gestion de la propriété privée y existent. À cause de l'encadrement particulier des droits de propriété dans les sociétés libérales capitalistes, le travail normatif de définition des besoins de l'homme capitaliste est le fait plutôt des spécialistes de mercatique ou de relations publiques. Or ces spécialistes, investis par le droit libéral du pouvoir de sculpter l'homme capitaliste, et travaillant pour diriger la 
consommation et encadrer l'utilisation de la propriété privée dans les sociétés libérales, ne sont-ils pas aussi efficaces dans leur capacité de suggérer et définir les normes de la société, et enfin de promouvoir des conditions menant aux monopoles et causes d'injustices majeures, que les planificateurs soviétiques assis dans les bureaux du Gosplan à Moscou?

On pourrait être tenté d'utiliser ce livre comme simple ouvrage de référence. Mais seule une lecture méticuleuse rendra justice à la richesse de l'analyse et aux strates multiples de signification de cette réflexion dense. Ainsi, parlant de la collectivisation, Aurore Chaigneau affirme que le processus «ne s'est pas accompagné d'une véritable mise en commun des moyens, mais a conduit à l'isolement des hommes» (p. 26). La collectivisation fut une série d'événements traumatiques pour les populations agraires, menant dans certains endroits à l'apparition d'une mentalité de collectivité et de communauté pour plusieurs générations de Soviétiques. Cette affirmation ne trouve tout son sens que plus loin dans le texte. L'auteur explique sa conception du phénomène d'isolement de l'individu dans le cadre d'un droit soviétique qui ne régule pas tant les relations entre individus que la relation entre l'individu et l'État. Pour cette raison, et bien qu'elle nous offre un livre dense de plus de 600 pages, d'où le lecteur doit extraire les arguments enfouis dans une masse d'informations, l'ouvrage demande une lecture attentive.

Cette tapisserie historique riche et complexe éclaire un aspect particulier du développement de la propriété dans un contexte trop souvent interprété à la lumière des expériences occidentales et non dans son contexte historique et politique. Le Droit de propriété en mutation intéressera un large public, qui ne se limite pas aux seuls spécialistes du droit en Russie. L'analyse du développement de la terminologie du droit en Russie sera particulièrement utile aux férus d'études comparatives du droit (un index plus détaillé, incluant la couverture minutieuse des débats entre penseurs russes, donnerait sa juste valeur à l'ouvrage). Les lecteurs de sciences sociales rencontreront là une spécialiste du droit qui n'est pas étrangère aux approches qui valorisent l'attention aux sens sociaux de la propriété. L'ouvrage intéressera également les spécialistes du domaine des politiques publiques ainsi que les universitaires. In fine on s'interroge sur les possibilités d'appropriation et de gestion des ressources naturelles, contribuant ainsi aux débats contemporains en Russie, en France... et ailleurs. 